\title{
Effect of Myocardial Fiber Direction on Epicardial Activation Patterns
}

\author{
Lindsay C Rupp ${ }^{1,2,3}$, Wilson W Good ${ }^{1,2,3}$, Jake A Bergquist ${ }^{1,2,3}$, \\ Brian Zenger ${ }^{1,2,3,4}$, Karli Gillette ${ }^{5}$, Gernot Plank ${ }^{5}$, Rob S MacLeod ${ }^{1,2,3}$ \\ ${ }^{1}$ Scientific Computing and Imaging Institute, University of Utah, SLC, UT, USA \\ ${ }^{2}$ Nora Eccles Cardiovascular Research and Training Institute, University of Utah, SLC, UT, USA \\ ${ }^{3}$ Department of Biomedical Engineering, University of Utah, SLC, UT, USA \\ ${ }^{4}$ School of Medicine, University of Utah, SLC, UT, USA \\ ${ }^{5}$ Institute of Biophysics, Medical University of Graz, Graz, Austria
}

\begin{abstract}
Fiber structure governs the spread of excitation in the heart, however, little is known about the effects of physiological variability in the fiber orientation on epicardial activation. To investigate these effects, we used computer simulation to compare ventricular activation sequences initiated from stimulus sites at regularly spaced depths within the myocardium under varying rule-based fiber ranges. We compared the effects using four characteristics of epicardial breakthrough (BKT): location, area, shape (calculated via the axis ratio of a fitted ellipse), and orientation. Our results showed changes in the BKT characteristics as pacing depth increased, e.g., the area increased, the shape became more circular, and the orientation rotated counterclockwise, regardless of the fiber orientation. Furthermore, the maximal differences in epicardial activation from a single pacing site for location, area, axis ratio, and orientation were $1.2 \mathrm{~mm}, 74 \mathrm{~mm}^{2}, 0.16$, and $26^{\circ}$, respectively. Our results suggest that variability in fiber orientation has a negligible effect on the location, area, and shape of the BKT, while fluctuations were observed in the $B K T$ orientation in response to the fiber fields, especially for epicardial stimulation sites. Our results suggest the fiber field orientation plays only a minor role in activation simulations of ectopic beats.
\end{abstract}

\section{Introduction}

The spread of excitation in the heart is influenced by the underlying myocardial fiber structure [1] but the impact of variability in fiber orientation remains unknown. Although previous studies have shown physiological variability in fiber orientation up to $15^{\circ}$ on the epicardial and endocardial surfaces, the impact of such variability on electrical propagation is not well understood. [2] Previous reports have investigated only nonphysiological fiber fields, such as a constant fiber orientation throughout the myocardium. [3] Knowledge of how physiological changes in fiber orientation alter electrical propagation is necessary to interpret epicardial and torso surface activation patterns and use them clinically to localize sources of arrhythmias.

We hypothesized that physiological changes in myocardial fiber orientation could directly affect characteristics of the primary breakthrough (BKT) on the epicardium. To explore this hypothesis, we created an image-based ventricular model derived from a single canine heart and then applied seven rule-based fiber fields that were varied to have transmural fiber ranges from 90 to $150^{\circ}$. [4] Eikonal activation simulations were implemented using the Cardiac Arrhythmia Research Package (CARP) [5] via a single point stimulus and nine pacing depths in the left ventricle free wall. To characterize the relation between myocardial fiber orientation, stimulation depth, and epicardial activation, we investigated the location, size, shape, and orientation of the BKT. Our results showed that physiological variations in fiber orientation have only a negligible effect on the location, size, and shape of the BKT, but do produce responses in the BKT orientation.

\section{Methods}

\subsection{Simulation Model}

For our study, an anatomical bi-ventricular geometric model was derived from MRI scans of an explanted canine heart and represented using the Uniform Ventricular Coordinates (UVC). [6,7] The resulting mesh had an average edge length of $660 \mu \mathrm{m}$. Within the mesh, we used the Python-based framework carputils [4] to implement a rule-based fiber algorithm, which assumed a linear rotation of the fiber angle $\alpha$ and imbrication angle $\beta$ from the endocardium to the epicardium. The computed myocardial fibers were varied to encompass a large range of physio- 
logical values with symmetric bounds (Table 1), resulting in a total of seven fiber fields. These fields were also defined using an imbrication fiber orientation of $-65^{\circ}$ on the endocardium and $25^{\circ}$ on the epicardium. Various surfaces required to impose Dirichlet boundary conditions within the mesh were automatically extracted using meshtool, and subsequent Laplacian-Dirichlet solutions were computed within the CARPentry simulator $[5,8]$.

Table 1. The seven combinations of fiber fields. The fields had endocardial fibers that ranged from $45^{\circ}$ to $75^{\circ}$ in increments of $5^{\circ}$, with a corresponding set of epicardial fiber orientations that had symmetrical orientations, resulting in a variable range of fiber directions from 90 to $150^{\circ}$. All angles were relative to a horizontal at $0^{\circ}$.

\begin{tabular}{|c|c|c|}
\hline \hline $\begin{array}{c}\text { Fiber Field } \\
\text { A }\end{array}$ & $\begin{array}{c}\text { Endo. Orientation } \\
45^{\circ}\end{array}$ & $\begin{array}{c}\text { Epi. Orientation } \\
-45^{\circ}\end{array}$ \\
\hline B & $50^{\circ}$ & $-50^{\circ}$ \\
\hline C & $55^{\circ}$ & $-55^{\circ}$ \\
\hline D & $60^{\circ}$ & $-60^{\circ}$ \\
\hline E & $65^{\circ}$ & $-65^{\circ}$ \\
\hline F & $70^{\circ}$ & $-70^{\circ}$ \\
\hline G & $75^{\circ}$ & $-75^{\circ}$ \\
\hline
\end{tabular}

We performed simulations of the spread of activation for every fiber model constructed and a set of pacing sites spaced equally along a virtual transmural needle. For efficiency, we used an Eikonal-based depolarization sequence with the Cardiac Arrhythmia Research Package (CARP). The virtual pacing needle was placed within the left ventricle free wall and pacing was induced from nine depths spaced at approximately $1.4 \mathrm{~mm}$. The conduction velocities of the model were tuned to achieve total activation times similar to those from our experiments. The longitudinal conduction velocities were determined for epicardial, mid-myocardial, and endocardial stimulation sites as 96 , 102 , and $97 \mathrm{~cm} / \mathrm{s}$, respectively. The transverse and sheet conduction velocities were defined as $2 / 3$ and $1 / 3$ of the longitudinal value, respectively.

\subsection{Characterization Metrics}

Four metrics were implemented to characterize changes of the BKT pattern in response to different pacing depths and fiber fields: (1) location of earliest activation, (2) area within the BKT isochrone, and (3) shape and (4) orientation of BKT isochrone. The BKT was defined as the isochrone corresponding to $10 \%$ of the epicardial total activation time. To determine the shape and orientation of the BKT isochrone, its three-dimensional points were projected onto a plane fit to the region. A set of conic equations was used to fit an ellipse to these projected points. The BKT shape was then determined via the ratio of the major and minor axes of the fitted ellipse. In order to calculate the BKT orientation, a reference axis was calculated as follows: the long axis of the heart was approximated via the global gradient of the UVC Z coordinate. Relative to this coordinate systems, we selected an orthogonal plane to contain the short axis of the heart and the orientation of the BKT was calculated by determining the angle between the major axis of the fitted ellipse and the short axis (Figure 1).

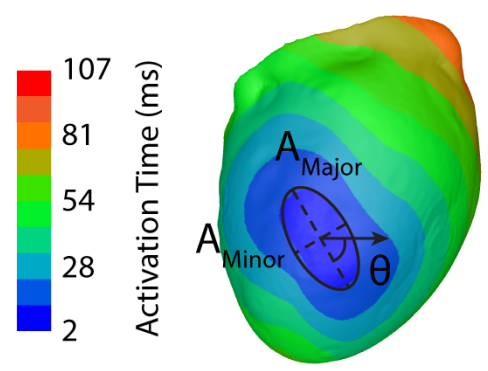

Figure 1. Characterization metrics of the BKT site. The location, area, shape and orientation of the BKT site were calcualted. An ellipse was fit to the BKT site and the ratio of the resulting major and minor axes was used to describe the shape of the BKT. The angle $(\theta)$ between the major axis and the short axis of the heart was its orientation.

\subsection{Variability Quantification}

We computed the area, shape, and orientation of the BKT for each stimulation site and fiber orientation. To quantify variability in the location of the BKT site, pairwise distances were calculated for all fiber field combinations within the same stimulation site, resulting in 21 values for each stimulation site, from which we computed the average distance.

\section{Results}

The location of the BKT changed in response to the implemented fiber fields, with an average pairwise distance of $0.1 \pm 0.3 \mathrm{~mm}$ and a maximum pairwise distance of $1.2 \mathrm{~mm}$. The epicardial BKT area increased as a function of pacing depth (Figure 2), increasing from $140 \pm$ $2 \mathrm{~mm}^{2}$ for an epicardial pacing site to $650 \pm 20 \mathrm{~mm}^{2}$ for an endocardial pacing site (Figure 3A). Similarly, the ratio of the major and minor axes of the fitted ellipse increased with increasing pacing depth, indicating that the BKT became more circular than elliptical. The axis ratio increased from $0.49 \pm 0.02$ for an epicardial pacing site to $0.74 \pm$ 0.06 for an endocardial pacing site (Figure 3B). Fiber field $\mathrm{G}$, which had the largest fiber range, consistently had the largest area and axis ratio at each pacing site while fiber field A, the smallest fiber range, consistently had the smallest area and axis ratio. The orientation of the BKT progressively rotated counter-clockwise with increasing pacing depth. For example, the orientation of the BKT with respect to the short axis of the heart rotated from $-51 \pm$ 
$9^{\circ}$ for an epicardial pacing site to $4 \pm 2^{\circ}$ for an endocardial pacing site (Figure $3 \mathrm{C}$ ). The maximum standard deviation observed at a single pacing site across fibers was $31 \mathrm{~mm}^{2}$ for the area of the BKT at a pacing depth of $10.8 \mathrm{~mm}, 0.06$ for the axis ratio of the BKT at a pacing depth of $12.3 \mathrm{~mm}$, and $9^{\circ}$ for the orientation of the BKT at a pacing depth of $0.8 \mathrm{~mm}$.

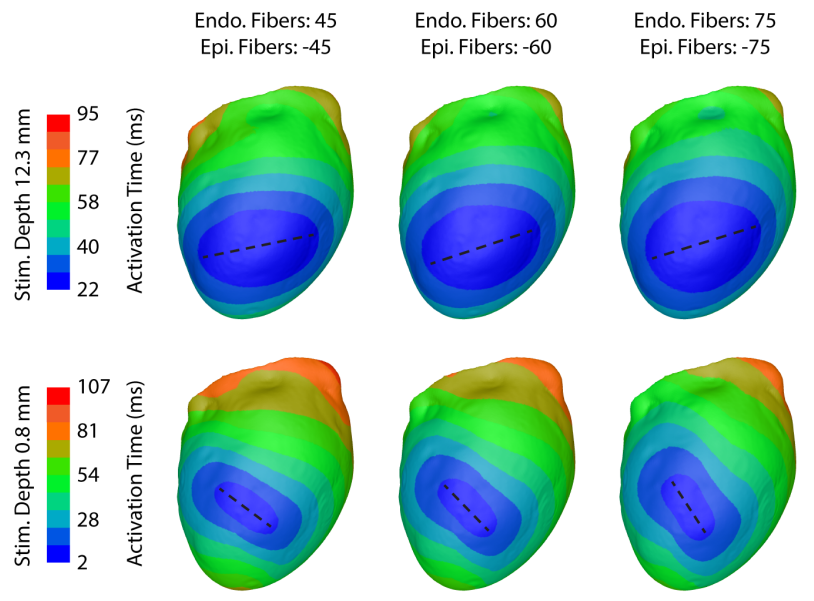

Figure 2. Activation time maps for increasing pacing depth and variable fibers. The darkest blue region is the selected BKT isocontour and the dashed black line is the orientation of the respective breakthrough sites. Top. Activation maps for an endocardial stimulation at a depth of $12.3 \mathrm{~mm}$ for endocardial to epicardial fiber ranges of 45 to $-45^{\circ}$ (left), 60 to $-60^{\circ}$ (middle) and 75 to $-75^{\circ}$ (right). Bottom. Activation maps for an epicardial stimulation at a depth of $0.8 \mathrm{~mm}$ for the same fiber fields listed previously.

\section{Discussion}

Cardiac electrical propagation is affected by myocardial fiber orientation, and the resulting shape of the epicardial breakthrough site is indicative of such influence. However, understanding the extent and degree of propagation changes in response to fluctuations within the fiber orientation is incomplete. Using ventricular heart simulations and rule-based fiber orientations, we captured breakthrough features after modeling variations in the fiber orientation to match broadly physiological ranges (Table 1 ). The simulations were based on the Eikonal simplification, rather than a full bidomain formulation; however, we were concerned only with the activation sequence, for which their performances have been shown to be comparable [9]. To understand the effects of fiber field variations, four characteristics of the BKT were evaluated: the location, area, shape, and orientation. Our results match previous findings,[1] which suggested that as pacing depth increases, the area of the BKT increases, the shape becomes more circular, and the orientation rotates counterclockwise. We evaluated for the first time the response of these metrics
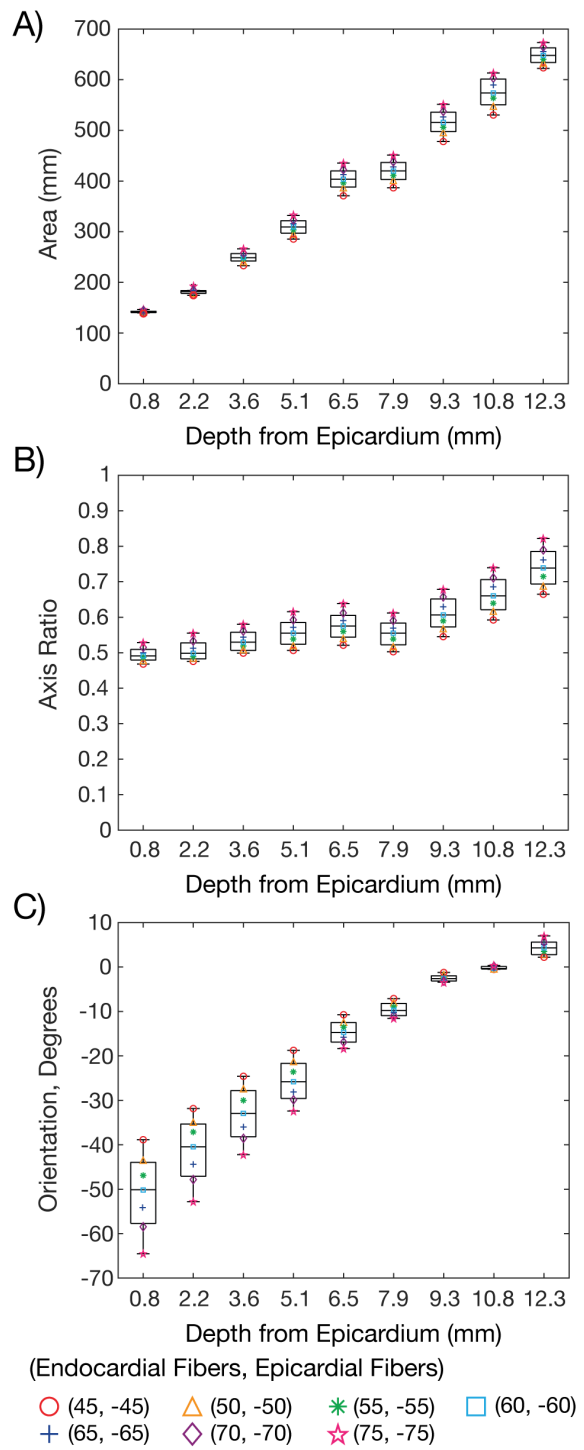

Figure 3. Variation in the calculated BKT site (A) area, (B) axis ratio, and $(C)$ orientation with respect to the seven implemented fiber fields. The legend depicted at the bottom denotes the different fiber field ranges implemented for each stimulation depth.

with respect to the fiber variations and found that the location, area, and shape of breakthroughs were minimally impacted. We also noted a similar lack of response for the BKT orientation following endocardial pacing. However, we noted that the different fiber fields had an effect on the BKT orientation following epicardial stimulation (Figure 3).

This response to epicardial or subepicardial pacing we hypothesize arises because the propagating wavefront travels through such a small number of fibers before reaching the epicardium. Therefore, the orientation of the BKT follows that of the superficial epicardial fibers so 
closely. However, endocardial pacing sites produce activations that must travel through the majority of the fiber field prior to reaching the epicardial surface, thus experiencing what appears to be a smoothing or averaging effect. Ultimately, endocardial pacing sites resulted in BKT orientations showing minimal variation with fiber orientation due to this smoothing.

We saw very little change in the location, area, and shape of the BKT in response to variation in fiber orientation. We hypothesize that the lack of change is because these metrics rely mostly on the transverse speed of propagation, which remains constant across variations in the longitudinal orientation of the fiber fields. Perhaps as a result of this behavior, current clinical techniques can determine the location of the site of origin of a premature ventricular beat, only to within a region of the heart greater than $10 \mathrm{~mm}^{2}$ in surface area, and thus our location variability of $0.2 \mathrm{~mm}$ is not a clinically relevant change. [10] Additionally, the maximum variability of the area and axis which we found to be $\pm 5 \%$ and $\pm 8 \%$, respectively, also do not present clinically relevant changes. A limitation of the study is that the results were generated from pacing within a small region in the left ventricular free wall. However, such a site has no apparent bias and is at least a promising starting point to build further results. Nevertheless, additional stimulation sites that encompass a broad range of effects will be evaluated in the future.

The fact that our models were based on rule-based fiber orientations represents both a potential limitation and a strength in terms of applications. While rule-based approaches have already shown their utility[4], they are not perfect. However, they represent the only means to impose a carefully controlled range of fiber orientations-subjectspecific heart models can never be so controlled. Even more relevant to the use of rule-based fibers is the finding that fiber orientation appears to be a very non-critical parameter of the models. Such findings provide even more confidence that rule-based approaches are easily accurate enough for simulation purposes and for clinical applications.

Future studies will focus on broadening the scope of this project to have a greater electrocardiographic impact. The simulations will be further applied to determine whether the small, but visible fluctuations in orientations on the epicardial activation isochrones fade when propagated to the torso. Such answers are critical to setting realistic limits to standard electrocardiography and to the potentially more accurate and elaborate electrocardiographic imaging (ECGI).

\section{Acknowledgments}

Support for this research came from the NIH / NIGMS Center for Integrative Biomedical Computing (www.sci.utah.edu/cibc), NIH NIGMS grants no. P41 GM103545 and R24 GM136986, and the Nora Eccles Treadwell Foundation for Cardiovascular Research.

\section{References}

[1] Taccardi B, Punske BB, Macchi E, MacLeod RS, Ershler PR. Epicardial and intramural excitation during ventricular pacing: effect of myocardial structure. American Journal of Physiology Heart and Circulatory Physiology 2008; 294(4):H1753-H1766.

[2] Lombaert H, Peyrat JM, Croisille P, Rapacchi S, Fanton L, Cheriet F, Clarysse P, Magnin I, Delingette H, Ayache N. Human atlas of the cardiac fiber architecture: study on a healthy population. IEEE transactions on medical imaging 2012;31(7):1436-1447.

[3] Muzikant AL, Henriquez CS. Bipolar stimulation of a three-dimensional bidomain incorporating rotational anisotropy. IEEE transactions on biomedical engineering 1998;45(4):449-462.

[4] Bayer JD, Blake RC, Plank G, Trayanova NA. A novel rule-based algorithm for assigning myocardial fiber orientation to computational heart models. Annals of biomedical engineering 2012;40(10):2243-2254.

[5] Vigmond E, Dos Santos RW, Prassl A, Deo M, Plank G. Solvers for the cardiac bidomain equations. Progress in Biophysics and Molecular Biology 2008;96(1):3-18.

[6] Zenger B, Good WW, Bergquist JA, Burton BM, Tate JD, Berkenbile L, Sharma V, MacLeod RS. Novel experimental model for studying the spatiotemporal electrical signature of acute myocardial ischemia: a translational platform. Physiological Measurement 2020;41(1):15002. ISSN 13616579.

[7] Bayer J, Prassl AJ, Pashaei A, Gomez JF, Frontera A, Neic A, Plank G, Vigmond EJ. Universal ventricular coordinates: A generic framework for describing position within the heart and transferring data. Medical image analysis 2018;45:83-93.

[8] Neic A, Gsell MA, Karabelas E, Prassl AJ, Plank G. Automating image-based mesh generation and manipulation tasks in cardiac modeling workflows using meshtool. SoftwareX 2020;11:100454.

[9] Neic A, Campos FO, Prassl AJ, Niederer SA, Bishop MJ, Vigmond EJ, Plank G. Efficient computation of electrograms and ecgs in human whole heart simulations using a reaction-eikonal model. Journal of computational physics 2017;346:191-211.

[10] Zhou X, Fang L, Wang Z, Liu H, Mao W. Comparative analysis of electrocardiographic imaging and ecg in predicting the origin of outflow tract ventricular arrhythmias. Journal of International Medical Research 2020; 48(3):0300060520913132.

Address for correspondence:

Name: Lindsay Cecala Rupp

Full postal address: SCI Institute, University of Utah, 72 Central Campus Dr, Salt Lake City, UT 84112

E-mail address: lindsay.rupp@utah.edu 